\title{
Uso de profilaxis antibiótica en el taponamiento nasal, una revisión actualizada
}

\author{
Antibiotic prophylaxis for nasal packing, an updated review
}

\author{
Valeria Sepúlveda C. ${ }^{1}$, Phoebe Ramos $Y^{2}$, Antonia Lagos $V^{2,3}$, Matías Willson E. ${ }^{4,5}$
}

${ }^{1}$ Escuela de Medicina, Pontificia Universidad Católica de Chile. Santiago, Chile. 2Departamento de Otorrinolaringología, Pontificia Universidad Católica de Chile. Santiago, Chile.

${ }^{3}$ Hospital El Carmen - Dr. Luis Valentín Ferrada, Servicio de Metropolitano Central. Santiago, Chile. ${ }^{4}$ Hospital Dr. Sótero del Río, Servicio de Salud Metropolitano Sur Oriente. Santiago, Chile.

${ }^{5}$ Hospital Padre Hurtado, Servicio de Salud Metropolitano Sur Oriente. Santiago, Chile.

Los autores declaran no tener conflictos de interés.

Recibido el 27 de julio de 2020. Aceptado el 7 de octubre de 2020.

Correspondencia: Matías Willson E.

Centro de Diagnóstico Terapéutico, Servicio de Otorrinolaringología, Av. Concha y Toro 3459, Puente Alto. Santiago, Chile Email: matias.willson@gmail.com

\section{Resumen}

El uso de profilaxis antibiótica en el taponamiento nasal es una práctica ampliamente usada por el eventual riesgo de síndrome de shock tóxico o complicaciones infecciosas locales. En los últimos años, se ha cuestionado su real impacto tomando en cuenta los riesgos asociados al uso de antibióticos y la baja o casi nula incidencia de las complicaciones infecciosas. Se realizó una revisión exhaustiva de la literatura sobre el uso de profilaxis antibiótica en taponamiento nasal. Se observó que no existían diferencias estadísticamente significativas en la incidencia de complicaciones infecciosas y no hubo reportes de síndrome de shock tóxico en ninguno de los grupos con y sin profilaxis antibiótica. No hubo diferencias significativas en los cultivos de los pacientes con o sin antibióticos en taponamiento nasal por epistaxis. El uso de profilaxis antibiótica en taponamiento nasal es una práctica cuestionable y se requieren más estudios al respecto.

Palabras clave: profilaxis, antibióticos, taponamiento nasal, seguridad.

\begin{abstract}
Antibiotic prophylaxis in nasal packing is a widely used practice due to the possible risk of toxic shock syndrome and infectious complications. Lately, its real impact has been questioned due to the associated risks with antibiotic use and low incidence of infectious complications. A comprehensive review of the literature on the use of antibiotic prophylaxis in nasal packing was performed. No statistically signifcant differences in the incidence of infectious complications and no reports of toxic shock syndrome were reported in any of the groups with and without antibiotic prophylaxis. There were no significant differences in the antibiotic cultures of patients with or without prophylactic antibiotics in nasal packing due to epistaxis. The use of antibiotic prophylaxis in nasal packing is a questionable practice and further studies are required.
\end{abstract}

Keywords: prophylaxis, antibiotics, nasal packing, safety.

\section{Introducción}

La epistaxis es una consulta frecuente tanto en el servicio de urgencia como en servicios de otorrinolaringología. Si bien no existen estadísticas nacionales, se ha reportado que hasta el $60 \%$ de la población sufre algún episodio de epistaxis en su vida, pero solo un $6 \%$ requeriría atención médica y un $0,16 \%$ hospitalización ${ }^{1}$. $\mathrm{Su}$ incidencia es mayor en edades pediátricas, antes de los 10 años, y a partir de los 35 a 40 años $^{1,2}$. En Estados Unidos (EE.UU.) entre
2009 y 2011, 1,2 millones de consultas al servicio de urgencias fueron por epistaxis, representando el $0,32 \%$ de todas las atenciones de urgencias ${ }^{1}$, y correspondiendo en el $90 \%$ a epistaxis anteriores ${ }^{3}$.

El taponamiento nasal anterior transitorio es generalmente utilizado como manejo conservador en epistaxis que no ceden con medidas locales conservadoras, como presión nasal o cauterización con nitrato de plata. Se utiliza para controlar el sangrado ${ }^{3}$ por un tiempo que puede variar entre 24 horas hasta 7 
días ${ }^{4}$. También es utilizado en cirugías nasales para hemostasia y prevención de hematomas septales y sinequias nasales, aunque su evidencia es controversial ${ }^{5}$. La mayoría de las veces este tipo de taponamiento se realiza con gasas o tórulas de algodón lubricados con ungüento (con o sin antibióticos). También existen otros materiales reabsorbibles y no reabsorbibles, como por ejemplo Merocel ${ }^{\circledR}$ (Medtronic Inc., Minneapolis, MN, EE. UU.), que es uno de los tapones de material no reabsorbible más comúnmente utilizados. Este corresponde a una esponja de acetato de polivinilo deshidratada comprimida, que aumenta de tamaño dentro de la cavidad nasal al rehidratarse con solución salina, comprimiendo los vasos sangrantes. Dentro de los tapones reabsorbibles se encuentra Nasopore ${ }^{\circledR}$ (Polyganics, Groningen, Holanda), uno de los más usados, o Posisep ${ }^{\circledR} \mathrm{X}$ (Hemostasis, Minnesota, EE. UU.), que se encuentra en el mercado chileno. Pueden ser de material completamente sintético, como el Nasopore ${ }^{\circledR}$, o en base a polímeros orgánicos, como el Posisep ${ }^{\circledR X}$. Este último está conformado por un polímero en base a chitina, que es un amino-polisacárido extraído del caparazón de crustáceos ${ }^{6}$, por lo que se debe tener precaución en los pacientes con alergia a los mariscos. Estos funcionan al absorber agua mientras sostienen el tejido circundante y presionan contra los vasos en la cavidad nasal, y luego se comienzan a disolver con el transcurso de los días (Posisep ${ }^{\circledR} \mathrm{X}$, en 5 a 10 días), y puede ser succionado desde la cavidad nasal después de varios días o tras la realización de lavados nasales ${ }^{7}$. Se recomienda el uso de materiales reabsorbibles en caso de pacientes con coagulopatías o uso de anticoagulantes para disminuir el riesgo de resangrado, como también en pacientes pediátricos, en los cuales el retiro y manipulación puede ser más difícil y traumático ${ }^{1}$.

La epistaxis posterior se origina secundaria a lesiones de las ramas de la arteria esfenopalatina lo que condiciona a un mayor volumen de sangrado y, por ende, mayor severidad. Existen múltiples opciones para su manejo, desde el uso de un taponamiento posterior con gasas con guía en la rinofaringe, o con una sonda Foley que se impacta sobre la rinofaringe. Se ha descrito el riesgo de generar un reflejo trigémino-cardiaco o de Kratschmer al impactar el taponamiento en la rinofaringe, presentándose como un episodio de hipotensión, bradicardia, o incluso asistolia ${ }^{1,8,9}$. Además, se han descrito lesiones de la piel, mucosa y cartílago producto de la presión sostenida, con deformidad estética asociada ${ }^{10}$. Fuera de lo antes descrito, este taponamiento se debe mantener entre 2 a 7 días, en general con una hospitalización para monitorización del paciente, y con el riesgo de hasta un $52 \%$ de resangrado a pesar de estas medidas ${ }^{1}$. Actualmente, la recomendación en estos casos es realizar un taponamiento anterior y posterior concomitante. Esto se puede realizar con material no reabsorbible, por ejemplo, Merocel ${ }^{\circledR}$ asociado o no a la instalación de una sonda Foley o bien, el uso de Rapid Rhino ${ }^{\circledR} 900$ (ArthroCare, Texas, USA) que posee un balón posterior que se impacta en la rinofaringe y un balón anterior posicionado en la cavidad nasal; o lo que se disponga de manera transitoria para resolución. En centros terciarios con equipo de otorrinolaringología, la terapia de elección es la ligadura o electrocauterio endoscópico de la arteria esfenopalatina, con una tasa de éxito de un $98 \%$ y riesgo de sangrado posoperatorio de un $3,4 \%^{1}$. Su alternativa es la embolización, utilizada desde 1974, con una tasa de éxito de un $87 \%$, con complicaciones menores en un 20\% (isquemia nasal transitoria, dolor temporofacial, cefalea, edema, claudicación mandibular e infecciones del sitio de punción sin necesidad de reintervención) y complicaciones mayores entre un $2,1 \%$ a un $3,8 \%$ (necrosis nasal, parálisis facial irreversible, amaurosis y accidente vascular encefálico $)^{1}$.

Una vez realizado el taponamiento anterior y/o posterior con detención del sangrado, se debe decidir si el manejo será ambulatorio u hospitalizado, de acuerdo al contexto de cada paciente. Se realiza educación sobre el manejo de los tapones en general, y en la mayoría de los casos se indica cobertura antibiótica durante la duración del taponamiento. Esto por el riesgo de infecciones locales como sinusitis u otitis media y por el riesgo de síndrome de shock tóxico (SST) ${ }^{3,11}$. Esta última es infrecuente, siendo reportada en 16,5 por cada 100.000 casos $^{3,11}$ posterior a la realización de cirugías de senos paranasales o septales ${ }^{12,13}$. Sin embargo, actualmente existe escasa evidencia que soporte esta recomendación y se deben ponderar 
los potenciales riesgos y beneficios asociados al uso de antibióticos sistémicos ${ }^{11}$. En los últimos años nos hemos visto enfrentados a un aumento en la resistencia antibiótica, que se ha atribuido a la prescripción inapropiada y el uso masivo de antibióticos ${ }^{14,15}$. Asimismo, se deben considerar los efectos adversos asociados a su uso $^{3}$. El objetivo de esta revisión es presentar la evidencia actual sobre el uso de profilaxis antibiótica en el taponamiento nasal como tratamiento de la epistaxis espontánea.

\section{Discusión}

El uso de antibióticos profilácticos posterior a la realización de un taponamiento nasal se debe al miedo del desarrollo de infección local (sinusitis aguda, otitis media) y especialmente el SST ${ }^{16,17}$, además algunos estudios describen mayor riesgo de readmisión y de recurrencia del sangrado ${ }^{18}$.

El SST fue inicialmente descrito a principios de 1980 por Thomas y cols., y Toback y Fayerman, quienes reportaron casos que aparecen después de septoplastías y rinoseptoplastías, sobre todo asociados al uso de férulas nasales ${ }^{12}$. Posteriormente, en 1987 Jacobson y Kasworm reportaron una incidencia de 32 por cada 100.000 casos $^{16}$. Estos autores, describieron que los pacientes que desarrollaban SST eran portadores de Staphylococcus aureus, y que esta bacteria producía el SST a través de la toxina tipo 1. Además, para que el síndrome ocurriera tenía que existir una solución de continuidad en la mucosa nasal y los pacientes tenían que presentar déficit del anticuerpo antitoxina 116 . El cuadro clínico se caracteriza por la presencia de fiebre y erupciones cutáneas, asociado a shock séptico y falla multiorgánica ${ }^{17}$. Secundario a estas publicaciones, se comenzó a prescribir antibióticos profilácticos para prevenir el SST ${ }^{16}$. No obstante, hasta la fecha, solo existe un caso descrito en la literatura de un paciente con diagnóstico de leucemia mieloide aguda con trasplante de médula ósea que desarrolló un SST posterior a taponamiento anterior por epistaxis espontánea ${ }^{3}$. Estudios recientemente publicados reportan una prevalencia de SST de un $0,002 \%$ a $0,032 \%$ en pacientes posterior a cirugía de cavidades paranasales ${ }^{16}$.
Se ha descrito que el taponamiento nasal generaría cambios en la flora nasal, lo que podría llevar a un aumento en el riesgo de desarrollar SST e infecciones locales. Los resultados son controversiales. Algunos estudios evidencian que el uso de taponamiento nasal aumentaría la presencia de Staphylococcus aureus en la cavidad nasal, sin embargo, hay otros estudios que no demuestran cambios en la flora nasal ${ }^{16}$. Biswas y cols., no describen diferencias significativas con respecto al crecimiento bacteriano circundante al taponamiento nasal anterior en pacientes con tratamiento antibiótico profiláctico en comparación al grupo de pacientes sin antibióticos ${ }^{19,20}$. En estudios similares, Derkay y cols. estudiaron pacientes que cursaron con taponamiento posterior con gasas impregnadas con antibiótico: en las gasas del grupo sin antibióticos sistémicos encontraron mal olor y estaban muy colonizadas por microorganismos gram negativos, en cambio, las de los pacientes con profilaxis sistémica no tenían olor y estaban ligeramente colonizadas por gram positivos ${ }^{21}$.

Por otro lado, los antibióticos profilácticos se indican para reducir el riesgo de infección local (sinusitis aguda y otitis media) asociada a taponamiento nasal ${ }^{16}$. Sin embargo, hasta la fecha existe escasa literatura sobre estas asociaciones ${ }^{16}$. Estudios han descrito que el taponamiento nasal generaría pérdida de los cilios nasales y cambios en la mucosa nasal (congestión e hipervascularización) ${ }^{16}$. Es por esto que se presume que el uso de taponamiento nasal altera el barrido mucociliar, bloquea el drenaje de los senos paranasales y, además, actuaría como un reservorio bacteriano ${ }^{16}$. En la revisión sistemática realizada por Lange y cols. describen una proporción total de sinusitis e infecciones locales de aproximadamente un $8,2 \%{ }^{16}$. En el metaanálisis realizado por Tran y cols. describen que solo un $0,8 \%$ de los pacientes presentaron una infección clínicamente significativa asociada al uso de taponamiento anterior ${ }^{3}$. Por otro lado, con respecto al desarrollo de abscesos septales e infecciones locales posterior a septoplastía, se ha descrito una prevalencia entre un $0,48 \%$ a $12 \%{ }^{16}$.

En la revisión sistemática escrita por Lange y cols., describen que los estudios incorporados en su revisión no recomendaban el uso de antibióticos profilácticos para el taponamien- 
to nasal. De un total de 3.125 pacientes sólo uno desarrolló una infección local. No hubo diferencias significativas entre pacientes que recibieron antibióticos en comparación a los que no recibieron. Sin embargo, esta revisión presenta limitaciones, como: la mayoría de los pacientes incluidos tuvo un taponamiento nasal luego de una septoplastía; algunos estudios utilizaron antibióticos tópicos en el taponamiento nasal o luego de su retiro; los estudios incluidos eran heterogéneos en cuanto a la duración del taponamiento (12 a más de 72 horas), siendo difíciles de comparar. Cabe destacar, que ningún estudio logró diferencias estadísticamente significativas para recomendar o no el uso de antibióticos en pacientes con taponamiento nasal ${ }^{16}$.

A pesar de que la evidencia actual es escasa y no concluyente con respecto a los beneficios en relación al uso de antibióticos en taponamiento nasal, en la práctica clínica persiste su uso $^{18}$. Encuestas realizadas en países europeos evidencian que más de un $50 \%$ de los clínicos, prescribe antibióticos profilácticos en pacientes con taponamientos nasales, tanto para el manejo de epistaxis como posterior a cirugía nasal ${ }^{16}$. Biswas y cols. describen que un $22 \%$ de los otorrinolaringólogos en Inglaterra no utiliza antibióticos de forma rutinaria, mientras que un 5\% utiliza taponamiento en todos los pacientes, $37 \%$ prescribe antibióticos en pacientes con taponamiento nasal de más de 24 horas de duración, y sólo $28 \%$ prescribe antibióticos en pacientes con taponamiento nasal de más de 48 horas de duración ${ }^{17,22}$. Con respecto a la duración del taponamiento nasal, un $43 \%$ mantiene el taponamiento por 5 días, un $14 \%$ por 7 días y un $6 \%$ no presentó preferencias en la duración ${ }^{22}$. Con respecto al tipo de antibiótico, un 73\% utiliza amoxicilina con ácido clavulánico, 17\% sólo amoxicilina y un $10 \%$ cefalosporinas ${ }^{22}$. Estudios realizados en EE. UU. reportan una incidencia de profilaxis antibiótica en el servicio de urgencia de un $61 \%$ en el año 2001, que ha disminuido a un $46 \%$ entre los años 2012 a $2016^{3}$.

Murano y cols. no encontraron signos de infección en ninguno de los pacientes, independiente del uso de antibióticos. Describen que aproximadamente el 50\% de los médicos en la urgencia indican profilaxis antibiótica, explican que esto se puede deber a la escasez de estudios potentes que demostrarían una falta en el beneficio de esta práctica clínica. Sugieren la implementación de algoritmos de tratamiento para estandarizar las prácticas clínicas y estratificar el riesgo en pacientes que se benefician de un tratamiento antibiótico profiláctico ${ }^{18}$. Concluyen que el tratamiento antibiótico profiláctico sería innecesario y no lo recomienda.

Pepper y cols. realizaron un estudio prospectivo de pacientes con diagnóstico de epistaxis con indicación de taponamiento nasal derivados a un centro terciario de otorrinolaringología. Un total de 78 pacientes utilizaron antibiótico oral (amoxicilina con ácido clavulánico $625 \mathrm{mg}$ tres veces al día) por 5 días, y en caso de alergia a la penicilina utilizaron claritromicina $500 \mathrm{mg}$ dos veces al día. Compararon este grupo con otro grupo de pacientes con epistaxis espontánea y taponamiento anterior sin uso de antibióticos como profilaxis ${ }^{17}$. Evaluaron la presencia de complicaciones asociadas (SST, infección sinusal y otitis media), sin encontrar diferencias significativas, por lo tanto, no recomiendan el uso de profilaxis antibiótica en pacientes con taponamiento anterior secundaria a epistaxis espontánea ${ }^{17}$.

Es importante conocer y sopesar los riesgos asociados al uso inadecuado de antibióticos, ya que se asocian a efectos adversos potencialmente graves, mayores costos y mayor resistencia antibiótica ${ }^{16}$. El Centers for Disease Control and Prevention (CDC) reporta que hasta un 30\% de las prescripciones de antibióticos realizadas por médicos son innecesarias ${ }^{3}$. Además, pueden presentar una serie de reacciones adversas al uso de penicilinas con una prevalencia aproximada de $2,2 \%$ a $5,1 \%{ }^{16,18}$; síndrome de Stevens-Johnson y alteraciones gastrointestinales ${ }^{18}$. Asimismo, el desarrollo de infección por Clostridium difficile (CD) es responsable de un $10 \%$ a $25 \%$ de las diarreas asociadas al uso de antibióticos ${ }^{3,16}$. Tradicionalmente la infección por CD se ha asociado al uso de clindamicina, pero actualmente, ha aumentado su incidencia asociada con el uso de penicilinas y cefalosporinas, que son dos antibióticos frecuentemente utilizados en taponamiento nasal ${ }^{16}$. Se describe que 1 de cada 80 pacientes con taponamiento nasal anterior y/o posterior que recibe profilaxis antibiótica 
reporta infección por $\mathrm{CD}^{3}$. Es posible que los riesgos asociados al uso de antibióticos superen los beneficios considerando las posibles complicaciones relacionadas ${ }^{11}$.

Actualmente, no existen guías clínicas o estudios clínicos randomizados controlados que aconsejen el uso de terapia antibiótica como profilaxis $^{19}$. La evidencia está basada en estu- dios retrospectivos pequeños, observacionales y no randomizados ${ }^{11}$. Además, pareciera no existir consenso sobre la duración del taponamiento y del tratamiento antibiótico asociado. Los estudios evaluados en este trabajo no presentan evidencia potente que avale o desestime completamente el uso de profilaxis antibiótica asociado a taponamiento nasal (Tabla 1). Es

Tabla 1. Resumen de estudios sobre profilaxis antibiótica en taponamiento nasal

\begin{tabular}{|c|c|c|c|c|c|c|}
\hline Estudio & Diseño & Población & $\mathbf{N}$ & Edad (años) & Grupos e intervención & Resultados \\
\hline $\begin{array}{l}\text { Pepper } \\
\text { y cols. }{ }^{17}\end{array}$ & $\begin{array}{l}\text { Prospectivo } \\
\text { no } \\
\text { randomizado }\end{array}$ & $\begin{array}{c}\text { Epistaxis } \\
\text { espontánea } \\
\text { con } \\
\text { taponamiento } \\
\text { nasal }\end{array}$ & 149 & $\begin{array}{l}\text { No } \\
\text { reportada }\end{array}$ & $\begin{array}{l}\text { Grupo } 1(\mathrm{~N}=78) \text { : } 5 \text { días de } \\
\text { amoxicilina ácido clavulánico } \\
625 \mathrm{mg} 3 \text { veces al día o } \\
\text { claritromicina } 500 \mathrm{mg} 2 \text { veces } \\
\text { al día } \\
\text { Grupo } 2(\mathrm{~N}=71) \text { : sin } \\
\text { profilaxis }\end{array}$ & $\begin{array}{l}\text { Ningún paciente } \\
\text { desarrolló sinusitis, SST u } \\
\text { otras complicaciones. }\end{array}$ \\
\hline $\begin{array}{l}\text { Murano } \\
\text { y cols. }{ }^{18}\end{array}$ & $\begin{array}{l}\text { Retrospectivo } \\
\text { (revisión de } \\
\text { fichas) }\end{array}$ & $\begin{array}{c}\text { Epistaxis } \\
\text { espontánea }\end{array}$ & 473 & $18-91$ & $\begin{array}{l}106 \text { pacientes con } \\
\text { taponamiento anterior. } \\
\text { Con antibióticos }(N=57) \\
\text { Sin antibióticos }(N=49)\end{array}$ & $\begin{array}{l}\text { Pacientes sin antibióticos } \\
\text { acudieron con mayor } \\
\text { frecuencia a control de } \\
\text { seguimiento ( } 79 \% \text { vs. } \\
49 \%) \text {. Sin complicaciones } \\
\text { infecciosas en ambos } \\
\text { grupos }\end{array}$ \\
\hline $\begin{array}{l}\text { Biggs } \\
\text { y cols. }{ }^{19}\end{array}$ & $\begin{array}{l}\text { Retrospectivo } \\
\text { (revisión de } \\
\text { fichas) }\end{array}$ & $\begin{array}{l}\text { Epistaxis } \\
\text { espontánea } \\
\text { con } \\
\text { taponamiento } \\
\text { anterior }\end{array}$ & 57 & $6-67$ & $\begin{array}{l}\text { Primera intervención }(\mathrm{N}=38 \text { ): } \\
\text { con profilaxis } \\
\text { Segunda intervención } \\
(\mathrm{N}=19) \text { : sin profilaxis si } \\
\text { taponamiento } \leq 48 \text { horas; } \\
5 \text { días de amoxicilina-ácido } \\
\text { clavulánico } 6253 \text { veces al } \\
\text { día o claritromicina } 500 \mathrm{mg} \\
2 \text { veces al día luego del retiro } \\
\text { de taponamiento si > } 48 \\
\text { horas } \\
\text { Total }(\mathrm{N}=57) \text { : neomicina } \\
\text { tópica luego del retiro }\end{array}$ & $\begin{array}{l}\text { Sin diferencias } \\
\text { significativas en } \\
\text { complicaciones } \\
\text { (descarga nasal, costras } \\
\text { nasales, dolor, sinusitis, } \\
\text { resangrado, readmisión). } \\
\text { Reducción de 58,2\% en } \\
\text { indicación de antibióticos } \\
\text { en grupo con profilaxis }\end{array}$ \\
\hline $\begin{array}{l}\text { Biswas } \\
\text { y cols. }{ }^{20}\end{array}$ & $\begin{array}{l}\text { Prospectivo } \\
\text { no } \\
\text { randomizado }\end{array}$ & $\begin{array}{c}\text { Epistaxis } \\
\text { espontánea }\end{array}$ & 28 & $\begin{array}{c}\text { No } \\
\text { reportada }\end{array}$ & $\begin{array}{l}\text { Taponamiento } \leq 24 \text { horas } \\
(\mathrm{N}=17) \text { : } \text { sin profilaxis } \\
\text { Taponamiento }>24 \text { horas } \\
\text { ( } \mathrm{N}=11) \text { : amoxicilina } \\
\text { clavulánico }\end{array}$ & $\begin{array}{l}\text { Sin evidencia } \\
\text { endoscópica de infección } \\
\text { nasal en ambos grupos. } \\
\text { Cultivos similares en } \\
\text { ambos grupos }\end{array}$ \\
\hline $\begin{array}{l}\text { Derkay } \\
\text { y cols. }{ }^{21}\end{array}$ & $\begin{array}{l}\text { Prospectivo } \\
\text { doble ciego }\end{array}$ & $\begin{array}{c}\text { Epistaxis } \\
\text { espontánea } \\
\text { con } \\
\text { taponamiento } \\
\text { posterior }\end{array}$ & 20 & $26-68$ & $\begin{array}{l}\text { Grupo placebo }(\mathrm{N}=10) \text { : } \sin \\
\text { profilaxis } \\
\text { Grupo antibióticos } \\
\text { endovenosos ( } \mathrm{N}=10) \text { : } \\
\text { cefazolina } 1 \mathrm{~g} \text { cada } 8 \text { horas } \\
\text { o clindamicina } 900 \text { mg cada } \\
8 \text { horas } \\
\text { Total }(\mathrm{N}=20) \text { : taponamiento } \\
\text { con vaselina con bacitracina }\end{array}$ & $\begin{array}{l}\text { Ningún paciente } \\
\text { desarrolló complicaciones } \\
\text { infecciosas (hemograma y } \\
\text { roentgenograma sinusal). } \\
\text { Crecimiento marcado } \\
\text { de gram negativos y mal } \\
\text { olor en el grupo placebo; } \\
\text { crecimiento de escasos } \\
\text { gram positivos y sin mal } \\
\text { olor en el grupo control }\end{array}$ \\
\hline
\end{tabular}


por esto que algunos estudios recomiendan no utilizar antibióticos en pacientes sin comorbilidades, sin embargo, Cohn y cols. sugieren considerar su uso en cierto grupo de pacientes como inmunodeprimidos o en pacientes con enfermedad cardiaca valvular ${ }^{11}$.

\section{Conclusión}

Luego de una revisión de la literatura disponible a la fecha, podemos concluir que la prescripción de profilaxis antibiótica en taponamiento nasal no tendría una influencia significativa en disminuir la incidencia de complicaciones clínicas, infecciosas ni de SST. Esto debido a que, en los grupos de pacientes sin antibióticos, la incidencia es prácticamente nula e idéntica a la de los grupos con profilaxis. Además, no se encontraron grandes diferencias entre los cultivos nasales de los grupos de estudio y control. Por lo anterior, es válido para el clínico cuestionarse la indicación de profilaxis antibiótica, y hacer de esta práctica muchas veces rutinaria, una basada en la evidencia. La evidencia hasta la fecha no justifica cabalmente su uso, y se debe evaluar caso a caso su indicación.

Se requieren más estudios al respecto, los cuales tengan una metodología estandarizada. Estudios futuros deben evaluar de forma prospectiva el uso de profilaxis antibiótica en una cohorte de pacientes con epistaxis anterior y/o posterior para verificar o refutar estas conclusiones.

\section{Bibliografía}

1. Tunkel DE, Anne S, Payne SC, et al. Clinical Practice Guideline: Nosebleed (Epistaxis). Otolaryngol Neck Surg. 2020;162(1_suppl):S1-S38. doi: $10.1177 / 0194599819890327$.

2. Retuert DR, Fuentealba DD, Bretón AI, Ricci LL, Nazar RS, Naser AG. Manejo de epistaxis posterior en el Hospital Clínico de la Universidad de Chile entre los años 2013 y 2016. Rev Otorrinolaringol Cir Cabeza Cuello. 2017;77(4):389-394. doi: 10.4067/s071848162017000400389.

3. Tran QK, Rehan MA, Haase DJ, Matta A, Pourmand A. Prophylactic antibiotics for anterior nasal packing in emergency department: A systematic review and meta-analysis of clinically-significant infections. Am
J Emerg Med. 2020;38(5):983-989. doi: 10.1016/j. ajem.2019.11.037.

4. Iqbal IZ, Jones GH, Dawe N, et al. Intranasal packs and haemostatic agents for the management of adult epistaxis: Systematic review. J Laryngol Otol. 2017;131(12):1065-1092. doi: 10.1017/ S0022215117002055.

5. Han JK, Stringer SP, Rosenfeld RM, et al. Clinical Consensus Statement: Septoplasty with or without Inferior Turbinate Reduction. Otolaryngol Head Neck Surg. 2015;153(5):708-720. doi: 10.1177/0194599815606435.

6. Hemostasis LLC. Posisep ${ }^{\circledR} \mathrm{X}$ Hemostat Dressing/ Intranasal Splint. 2019 [citado 26 de julio de 2020]. Disponible en: https://www.hemostasisllc.com/ products/posisepx-hemostat-dressing-intranasalsplint/.

7. Wang J, Cai C, Wang S. Merocel versus Nasopore for Nasal Packing: A Meta-Analysis of Randomized Controlled Trials. Lin SJ, ed. PLoS One. 2014;9(4):e93959. doi: 10.1371/journal.pone.0093959.

8. Jacobs J, Levine L, Davis H, Lefrak S, Druck N, Ogura J. Posterior packs and the nasopulmonary reflex. Laryngoscope. 1981;91(2):279-284. doi: 10.1288/00005537-198102000-00017.

9. Awasthi D, Roy TM, Byrd RP. Epistaxis and Death by the Trigeminocardiac Reflex: A Cautionary Report. Fed Pract. 2015;32(6):45-49.

10. Vermeeren L, Derks W, Fokkens W, Menger DJ. Complications of balloon packing in epistaxis. Eur Arch Oto-Rhino-Laryngology. 2015;272(10):30773081. doi: 10.1007/s00405-015-3529-5.

11. Cohn B. Are prophylactic antibiotics necessary for anterior nasal packing in epistaxis? Ann Emerg Med. 2015;65(1):109-111. doi: 10.1016/j. annemergmed.2014.08.011.

12. Jacobson JA, Kasworm EM. Toxic shock syndrome after nasal surgery. Case reports and analysis of risk factors. Arch Otolaryngol Head Neck Surg. 1986;112(3):329-332. doi: 10.1001/ archotol.1986.03780030093019.

13. Suzuki S, Yasunaga H, Matsui H, Fushimi K, Kondo $\mathrm{K}$, Yamasoba T. Complication rates after functional endoscopic sinus surgery: Analysis of 50,734 Japanese patients. Laryngoscope. 2015;125(8):1785-1791. doi: 10.1002/lary.25334.

14. García PC. Resistencia bacteriana en Chile. Rev Chil Infect. 2003;20(Supl 1):11-23.

15. Ávila F, Luppi M, Gaete P, Rivas A, Silva F, Olivares R. Cambios en las prescripciones y el consumo de antimicrobianos, luego de la implementación de recomendaciones de uso: experiencia en un hospital universitario. Rev Chil Infectol. 2019;36(3):253-264. doi: 10.4067/s071610182019000300253.

16. Lange JL, Peeden EH, Stringer SP. Are prophylactic systemic antibiotics necessary with nasal packing? 


\section{ARTÍCULO DE REVISIÓN}

A systematic review. Am J Rhinol Allergy. 2017;31(4):240-247. doi: 10.2500/ajra.2017.31.4454.

17. Pepper C, Lo S, Toma A. Prospective study of the risk of not using prophylactic antibiotics in nasal packing for epistaxis. J Laryngol Otol. 2012;126(3):257-259. doi: 10.1017/S0022215111003215.

18. Murano T, Brucato-Duncan D, Ramdin C, Keller S. Prophylactic systemic antibiotics for anterior epistaxis treated with nasal packing in the ED. Am J Emerg Med. 2019;37(4):726-729. doi: 10.1016/j. ajem.2018.12.056.

19. Biggs TC, Nightingale K, Patel NN, Salib RJ. Should prophylactic antibiotics be used routinely in epistaxis patients with nasal packs. Ann R Coll Surg Engl. 2013;95(1):40-42. doi: 10.1308/003588413X13511609954734.

20. Biswas D, Mal RK. Are systemic prophylactic antibiotics indicated with anterior nasal packing for spontaneous epistaxis? Acta Otolaryngol. 2009;129(2):179-181. doi: 10.1080/00016480802043964.

21. Derkay CS, Hirsch BE, Johnson JT, Wagner RL. Posterior nasal packing. Are intravenous antibiotics really necessary? Arch Otolaryngol Head Neck Surg. 1989;115(4):439-441. doi: 10.1001/ archotol.1989.01860280037013.

22. Biswas D, Wilson H, Mal R. Use of systemic prophylactic antibiotics with anterior nasal packing in England, UK. Clin Otolaryngol. 2006;31(6):566-567. doi: 10.1111/j.1365-2273.2006.01336.x. 